\title{
A Near-Optimal Power Management Strategy for Rapid Component Sizing of Power Split Hybrid Vehicles with Multiple Operating Modes
}

\author{
Xiaowu Zhang Huei Peng Jing Sun
}

\begin{abstract}
In the design of hybrid vehicles, it is important to identify proper component sizes. When the search space of the design problem is large, exhaustive power management strategy such as dynamic programming (DP) is too time-consuming to be feasible. Instead, a near-optimal method that is orders of magnitude faster than DP is needed. One such near-optimal method is developed and presented in this paper. This method is applied to design an input-split hybrid vehicle utilizing a single planetary gear (PG). There are 6 possible input split configurations, and each configuration has up to 4 modes [1]. Based on the analysis of the efficiency of powertrain components of the four modes, and the "Power-weighted Efficiency" (PE) concept, we show that the computation time for each sizing problem can be reduced by a factor of 10,000 compared with the dynamic-programming based approach.
\end{abstract}

\section{INTRODUCTION}

The market of hybrid vehicles has been dominated by power-split configuration for years. More than $90 \%$ of the hybrid vehicles sold in the US in 2012 is power-split type [2]. The configuration of hybrid vehicle refers to the connection of powertrain components (namely the engine, 2 motors, and final drive) with the transmission, i.e., the three nodes of the planetary gear(s) (PG). The popularity of the power-split hybrids can be attributed to their capability to take advantages of both series and parallel configurations [3][4] and the high efficiency and compactness of the PG. Today's market leading power split designs, such as Toyota Prius, Ford Fusion and Chevy Volt, use a single planetary gear as the transmission device. Some other models, such as Lexus GS450h and Toyota Highlander Hybrid, use two planetary gears. There are also hybrid designs in combat vehicles [4] and in hybrid trucks [5].

Using a single $P G$ as the split device can produce 12 possible configurations ( 6 input-split and 6 output-split) and each configuration can have up to four useful modes [1]. Whereas two PGs can produce 1,152 combinations [4]. Some of the configurations are protected by patents (e.g., [6][7][8]). However, because of the size of the candidate pool, there are many potential designs not yet exploited. The focus of this paper is not on the identification and characterization of a

\footnotetext{
*Supported by CERC-CVC Foundation.

Xiaowu Zhang, Huei Peng, are with the Department of Mechanical Engineering, University of Michigan, Ann Arbor, MI, 48109

USA.(phone:734-647-9732;fax:734-764-4256;emails:xiaowuz@umich.edu, hpeng@umich.edu.

Jing Sun is with the Department of Naval Architecture \& Marine

Engineering, University of Michigan, Ann Arbor, MI, 48109 USA (phone:

734-615-8061, e-mail:jingsun@umich.edu)
}

particular configuration. Instead, we study a more fundamental and unconstrained problem: assuming all input-split single-planetary gear configurations are design candidates, we explore the full search space of configuration-sizing-control to find the best hybrid vehicle design, whereas the focus of the optimality is fuel economy. In the literature, powertrain sizing is mostly dominated by performance/drivability considerations [9][10][11]. When fuel economy is the main performance metric, sizing can be done through exhaustive search by iteratively solving an optimal energy management strategy for each of the sizing candidate. The control strategy can be solved using load leveling [12][13], Equivalent Consumption Minimization Strategy (ECMS)[14][15], the Pontryagin's Minimum Principle (PMP) $[16][17][18]$ or dynamic programming (DP) [19][20]. However, these strategies all have some drawbacks. Load leveling methods are not optimal, ECMS strategy is an instantaneous optimization method, DP suffers from heavy computation load, and PMP frequently have numerical convergence issues that are common for nonlinear two-point-boundary-value problems.

To mitigate these drawbacks, a rapid power management algorithm with the drive cycle information used in a statistical way is proposed. DP, as the only approach that guarantees global optimality over the problem horizon, will be used to calculate the optimal fuel economy. It serves as the benchmark to verify the performance of the proposed method.

This paper is organized as follows: In Section II, we illustrate the dynamics of the power-split system including its operation modes. In Section III, the rapid near-optimal power management algorithm is described. In Section IV, a case study to design the best input-split hybrid vehicle is presented with the results compared against results from DP. Finally in Section $\mathrm{V}$, the conclusions and future work are presented.

\section{DyNAMICS OF POWER SPLIT POWERTRAIN}

\section{A. Dynamics of Planetary Gear System}

A planetary gear (PG) system consists of a ring gear, a sun gear, and a carrier with several pinion gears. Its schematic together with its lever analogy are shown in Figure 1. A PG has 2 degrees of freedom (DOF), and the rotational speeds and accelerations of the three nodes (sun gear, ring gear, carrier) satisfy the constraint equation shown in Eq. (1).

$$
\omega_{s} S+\omega_{r} R=\omega_{c}(R+S)
$$


where the subscript $\mathrm{s}$ indicates the sun gear, $\mathrm{r}$ denotes the ring gear and $\mathrm{c}$ denotes the carrier. $\mathrm{S}$ and $\mathrm{R}$ are the radii of the sun gear and ring gear, respectively.
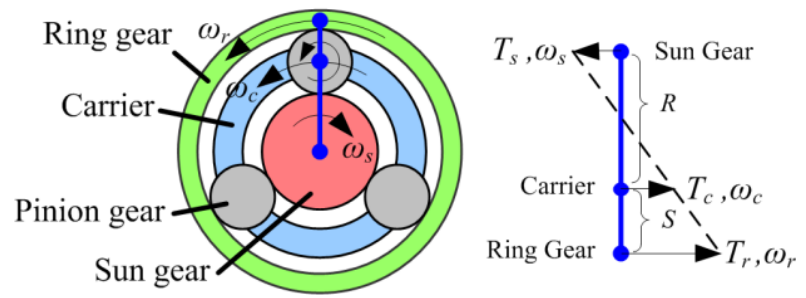

Figure 1. Planetary Gear and its Lever Analogy

The dynamics of a single planetary gear can be represented by Eq. (2), where $I_{(\cdot)}, T_{(\cdot)}$ are the inertia and torque of the components connected to the nodes and $F$ is the internal force between gear teeth.

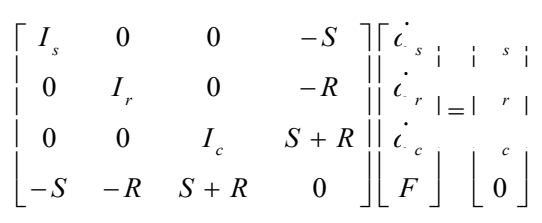

\section{B. Multiple Operating Modes}

In [1], it has been shown that when the engine is not directly connected to the output shaft and the two motor/generators (MGs) are not collocated, there are twelve possible configurations: 6 input-split configurations (one MG is connected to the output shaft) and 6 output-split configurations (one $\mathrm{MG}$ is connected to the engine). For a given configuration, clutches can be added to enable different operating modes. It is shown that at most 4 feasible modes can be achieved when 3 clutches are added [1]. The modes for one input-split configuration are shown in Figure 2.

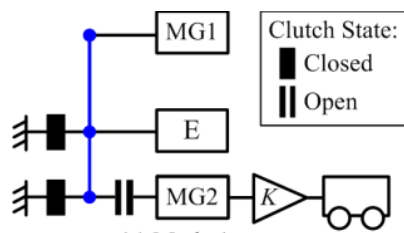

(a) Mode 1

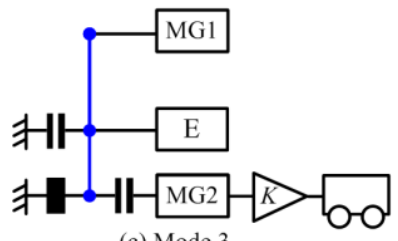

(c) Mode 3

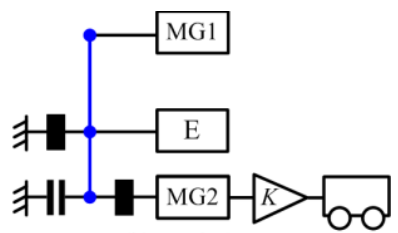

(b) Mode 2

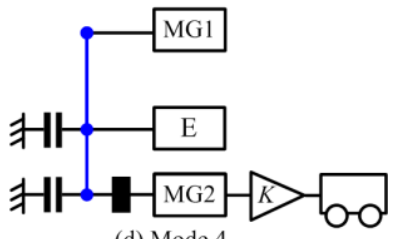

(d) Mode 4
Figure 2. All feasible modes for one input-split configuration

With rules stated in [21], the dynamic equations for the four modes are derived and presented below.

Mode $1\left(\mathrm{EV}_{1}\right)$ :

$$
\left(\frac{m r^{2}}{K^{2}}+I_{M G 2}\right) i_{\text {out }}-T_{\text {Load }}
$$

Mode $2\left(\mathrm{EV}_{2}\right)$ :

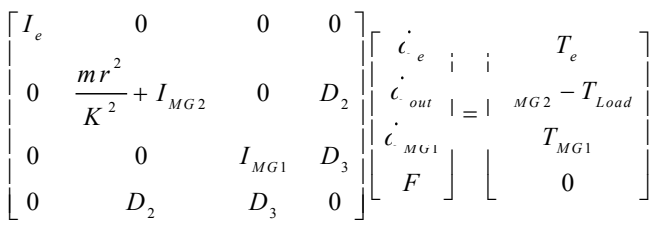

Mode 3 (Series):

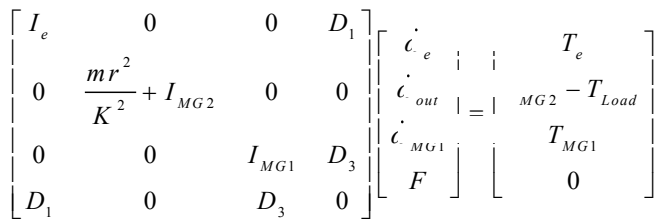

Mode 4 (Split):

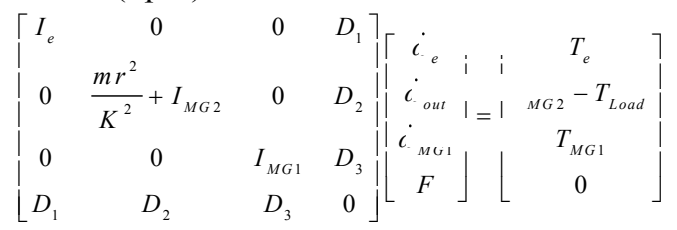

Where elements $\mathrm{D}_{1}, \mathrm{D}_{2}$ and $\mathrm{D}_{3}$ are permutations of $-R,-S$ and $\mathrm{R}+\mathrm{S}$, they denote the configuration of the hybrid system. More specifically, for each row, the entry is $-R$ if the component is connected to the ring gear; $-S$ if connected to the sun gear; and $R+S$ if connected to the carrier. $\dot{\omega}_{\text {out }}$ is the angular acceleration of the output node on the PG. $T_{\text {Load }}$ is defined in Eq. (7), where $K$ is the final drive ratio, and the three terms are for braking, rolling resistance and air drag.

$$
T_{\text {Load }}=\frac{1}{K}\left[T_{f b}+m g f_{r} R_{\text {tire }}+0.5 \rho A C_{d}\left(\frac{\omega_{\text {out }}}{K}\right)^{2} R_{\text {tire }}{ }^{3}\right]
$$

For the first three modes, the optimal dynamics are trivial because Modes 1 and 2 have only 1 degree of freedom while Mode 3 is a Series Mode. The Split Mode has two degree of freedom. Therefore, further analysis is needed to enable faster calculation for sizing, which is detailed in the following section.

\section{Split-Mode Analysis}

Since the Split Mode has 2 degrees of freedom, we can denote $\alpha$ as the ratio between $\dot{\omega}_{e}$ and $\dot{\omega}_{\text {out }}$. The vehicle behavior is then fully described by $\alpha$ and $\dot{\omega}_{\text {out }}$.

From Eq. (6), Eq. (8) can be obtained.

$$
\left[\begin{array}{l}
T_{M G 1} \\
T_{M G 2}
\end{array}\right]=\left[\begin{array}{l}
-\left[\left(I_{e} \frac{D_{3}}{D_{1}}+I_{M G 1} \frac{D_{1}}{D_{3}}\right) \alpha+I_{M G 1} \frac{D_{2}}{D_{3}}\right] i_{\text {out }} \\
T_{\text {Load }}+T_{e} \frac{D_{2}}{D_{1}}-\left(I_{e} \frac{D_{2}}{D_{1}} \alpha-\left(\frac{m r^{2}}{K^{2}}+I_{M G 2}\right)\right) i_{\text {our }}
\end{array}\right]
$$

As an example, when applying Prius' vehicle parameters, Eq.(8) becomes

$$
\left[\begin{array}{l}
T_{M G 1} \\
T_{M G 2}
\end{array}\right]=\left[\begin{array}{cc}
(0.14 \alpha-0.068) \dot{i}_{\text {out }} & T_{e} \\
T_{\text {Load }}-0.72 T_{e}-(0.13 \alpha-6.7) \dot{\epsilon}_{\text {out }}
\end{array}\right]
$$

With Eq. (8) or Eq. (9), $T_{M G 1}$ and $T_{M G 2}$ can be solved if $\dot{\omega}_{\text {out }}, \alpha, T_{e}$ and $T_{\text {load }}$ are given. A large $\alpha$ will quickly lead to engine and MG1 speed saturation. Therefore the value of $\alpha$ should be kept in a moderate range for normal driving. It can 
be seen that the demand torque will not be affected much within a practical range of $\alpha$, since inertia of other powertrain components are much smaller than the inertia of vehicle.

To enable fast sizing, the acceleration ratio $\alpha$ is assumed to be 1 (i.e. all components accelerate with the output-shaft at the same rate) in the remainder of this paper to reduce one degree of freedom in the split mode so that fast efficiency calculation in the next section is enabled.

\section{POWER-WEIGHTED EFFICIENCY ANALYSIS FOR RAPID SIZING}

Although global optimality is guaranteed by applying DP to explore the candidate design space, it is computationally demanding. For example, if 4 different design variables shown in Table 1 are explored, it will take more than 10 years to finish exhaustive search for a single configuration on a 4-core desktop available today. To overcome the time consuming drawback, several potential fast sizing methods have been considered.

Energy loss minimization is an effective way to optimize EV operation; however, it does not readily work when engine is running because of the large discrepancy between engine efficiency and motor efficiency. ECMS, a popular instantaneous optimization method, does not use cycle information, its mode shift decision thus only relies on the current power demand and state. Moreover, for split mode optimization, the MG efficiency is usually assumed to be constant which introduces error.

The Power-weighted Efficiency Analysis for Rapid Sizing (PEARS) in this paper is based on efficiency analysis of powertrain components. For a given drive cycle, we consider all possible vehicle speeds and load combinations and rearrange them into a 2D table. Components' torque and speed will be looped through, and the best power-weighted efficiency (PE) will be determined. With the selected vehicle operation, the Predicted Fuel Consumption (PFC) -- the fuel consumption calculated by the PEARS method-- is used to identify the optimal design choice. The process of PEARS is summarized in Figure 3. Note that in PEARS, battery open loop voltage and internal resistant is assumed to be constant.

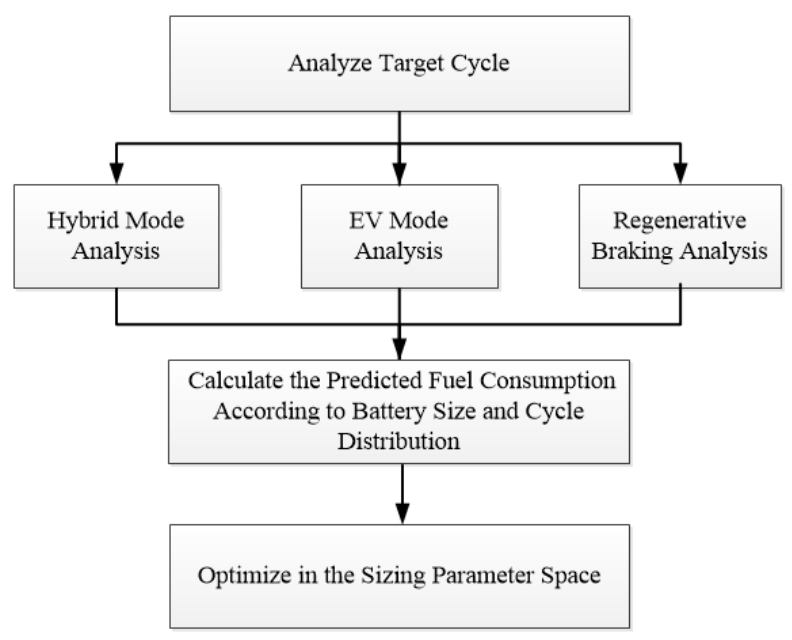

Figure 3. The flow chart of PEARS
The procedure is further delineated as follows:

Step 1:

First, the speed and acceleration data shown in Figure 4 for a given drive cycle is extracted. The extracted data is arranged into a two dimensional matrix (referred as Speed and Acceleration Cells, SACs), as plotted in Figure 5.
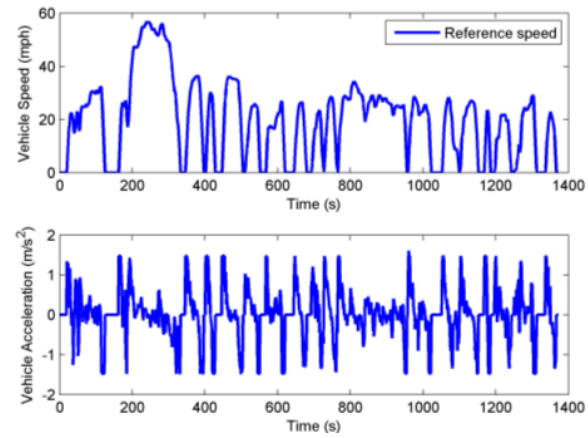

Figure 4. Speed and acceleration profile of FUDS cycle

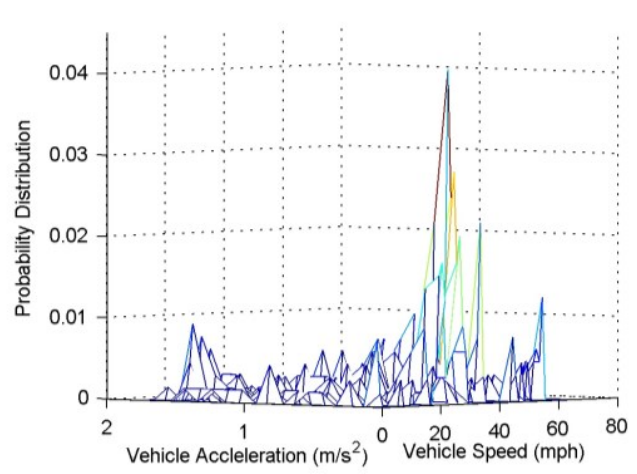

Figure 5. Probability distribution of the FUDS cycle when driving

Step 2.1(EV mode PE calculation):

The Power-weighted Efficiency (PE) for the two EV modes is defined in Eq. (10), where $P_{M G 1} i n, P_{M G 2}$ in,$\eta_{M G l}$, $\eta_{M G 2}$ are the power flow-in and the efficiency of MG1 and MG2, while $P_{E V \text { in }}$ is the power consumed by the electric system; $\eta_{\text {batt }}$ is the battery efficiency. For $E_{1}$ Mode, it is trivial to calculate the total efficiency since it is equal to the multiplication of the motor and battery efficiency. For $\mathrm{EV}_{2}$ Mode, all possible torque combination will be compared and the best total efficiency will be recorded. The mode with superior total efficiency will be considered as EV mode for each SAC. The optimal EV operation can be found by finding the maximum efficiency, denoted as $\eta_{E V}{ }^{*}$, with augments $\mathrm{T}_{\mathrm{MG} 1} *$ and $\mathrm{T}_{\mathrm{MG} 2} *$, as shown in Eq. (11).

$$
\begin{gathered}
\eta_{E V}=\frac{P_{M G 1 \_i n} \eta_{M G 1}+P_{M G 2_{-} i n} \eta_{M G 2}}{P_{E V \_i n}} \eta_{b a t t} \\
\left.\eta_{E V}{ }^{*}\right|_{i_{\text {out }, \text { out }}}=\eta_{E V}\left(T_{M G 1}{ }^{*}, T_{M G 2}{ }^{*}\right)
\end{gathered}
$$

\section{Step 2.2 (Hybrid model PE calculation):}

For each vehicle SAC, the vehicle load torque are calculated from Eq. (7). Together with Eq. (8), when assuming $\alpha=1$, the torque of MG1 and MG2 can be solved by looping through all possible engine torques. 
There are two power sources for hybrid mode: the engine and the battery. The power flows into the system can be divided into four parts: $P_{e_{1}}, P_{e_{2}}, P_{e_{3}}$ and $\underline{P}_{b a t t}$, where $P_{e_{-} l}$ is the fuel energy consumed corresponding to power goes to the battery; $P_{e_{-} 2}$ is the fuel energy consumed which goes through generator to motor; $P_{e_{3}}$ is the fuel energy consumed which flows to the final drive. $P_{e_{1} l}+P_{e_{2} 2}+P_{e_{3} 3}$ equal to the total fuel power. $P_{\text {batt }}$ is defined as the battery power consumed by the system. The power-weighted efficiency is the ratio between the utilized power and total consumed power as shown in Eq. (12).

Figure 6 describes the case when $P_{\text {batt }}<0$ and the two MGs act as one generator (when the power of MG is less than zero) and one motor (defined when the power of MG is greater than zero). When $P_{\text {batt }}>0$, the diagram is similar just with $P_{e_{-} l}=0$, and $\mu=1$.

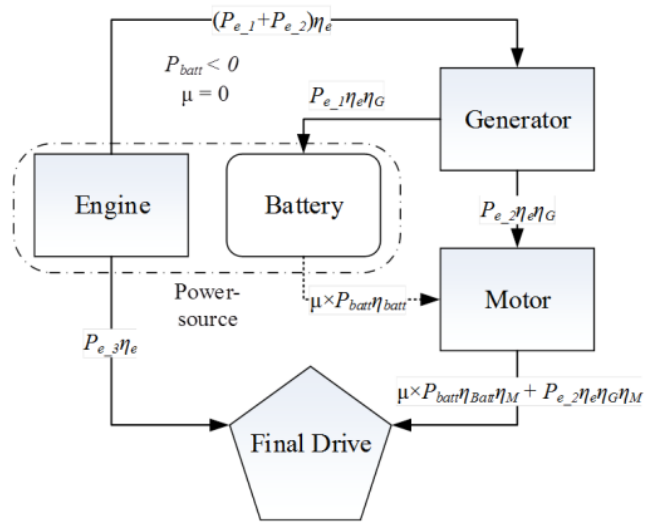

Figure 6. Power flow of the hybrid system when $\mathrm{P}_{\text {batt }}<0$

For the case that both MGs act as motors $\left(P_{M G l}>0\right.$, $P_{M G 2}>0$ ), we have $P_{e_{1}}=0, P_{e_{2}}=0$; when both MGs act as generators $\left(P_{M G 1}<0, \bar{P}_{M G 2}<0\right), \bar{P}_{e_{-} 2}=0, \underline{P}_{b a t t}=0$. Note that in Series Mode, $P_{e_{3} 3} \equiv 0$. At the end, the best efficiency of hybrid operations is denoted as $\eta_{\text {Hybrid }} *$ with the optimal augments $\omega_{\mathrm{e}}{ }^{*}$ and $\mathrm{T}_{\mathrm{e}}{ }^{*}$, as shown in Eq. (13).

$$
\begin{aligned}
& \eta_{H y b r i d}\left(\omega_{e}, T_{e}\right) \\
& =\frac{P_{e_{-} 1} \eta_{e} \eta_{M G 1} \eta_{\text {batt }}+P_{e_{-2} 2} \eta_{e} \eta_{M G 1} \eta_{M G 2}+P_{e_{-} 3} \eta_{e}+\underline{P}_{\text {batt }} \eta_{\text {batt }} \eta_{M G 2}}{P_{e}+\underline{P}_{\text {batt }}} \\
& \left.\eta_{\text {Hybrid }}{ }^{*}\right|_{i_{\text {out }, \text { out }}}=\eta_{p_{-} \text {hybrid }}\left(\omega_{e}{ }^{*}, T_{e}{ }^{*}\right)
\end{aligned}
$$

\section{Step 2.3 (PE for regenerative braking):}

When the vehicle decelerates, regenerative braking is applied and the EV Mode with the best efficiency is chosen following the process explained in Step 2.1. The calculation of PE follows Eq. (10) and Eq. (11), with $P_{E V_{-} \text {in }}$ defined as the mechanical power flows into the system.

Step 3:

Due to the fact that the engine efficiency is much lower than that of electric motors, a scaling strategy as Eq. (14) should be used to ensure fair comparison between EV and Hybrid efficiency. Where the $\max \left(\eta_{E V}\right)$ and $\max \left(\eta_{H y b r i d}\right)$ are the best possible EV and hybrid efficiency for all SACs.

Once the best PE for both EV and Hybrid Mode are calculated for each SAC, we first determine whether the vehicle is operating in the hybrid or EV mode for each SAC and then calculate the PFC.

$$
\bar{\eta}_{\text {Hybrid }}=\frac{\eta_{\text {Hybrid }} \times \max \left(\eta_{E V}\right)}{\max \left(\eta_{\text {Hybrid }}\right)}
$$

For the hybrid mode, since the engine efficiency is much lower than that of MG, battery assist will be preferred if we don't constrain the battery power. The maximum battery power is constrained to be negative to ensure- batteries are being charged. The information needed for the blended operation is listed below, which is shown in Figure 7:

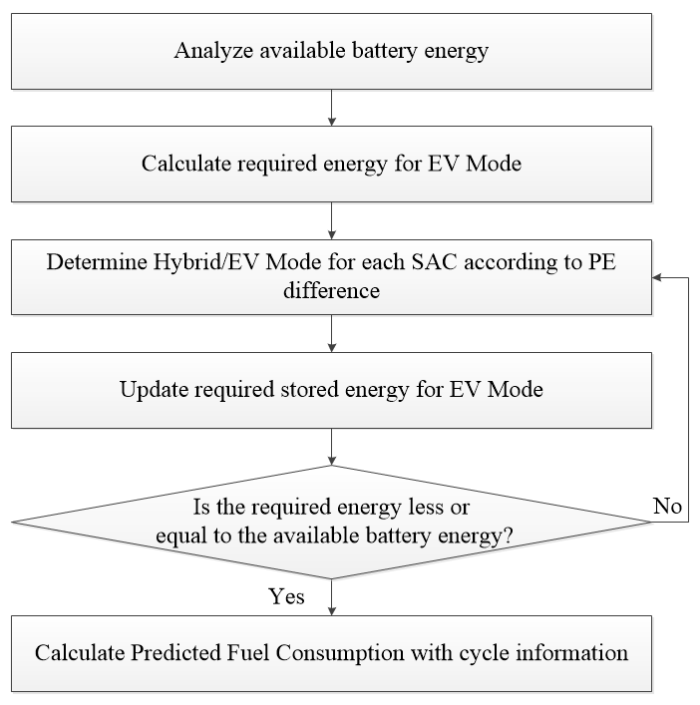

Figure 7. The flow chart of Step 3

i. Given the battery size, the total available battery energy $E_{a v}$ is calculated. For example, the available battery energy is $3.2 \mathrm{MJ}$ when the battery SOC drops from 0.55 to 0.43 .

ii. The PE difference $\bar{\eta}_{\text {Hybrid }}-\eta_{E V}$ is calculated for each SAC.

iii. The total required energy for the EV Mode is calculated from Eq.(15), where $N$ and $\mathrm{M}$ are the total number of SAC in driving and braking operation, $\Phi_{k}$ and $\Phi_{l}$ are the probability distribution of the $k_{\mathrm{th}}$ and $l_{t h}$ SAC, $T_{D}$ and $T_{B}$ is the total time duration when the vehicle is driving and braking, $P_{E V_{k}}$ and $P_{E V_{l}}$ are the battery power (negative when braking) with highest $\eta_{E V}$ at the $k t h$ and $l$ th $\mathrm{SAC}$ in the EV mode.

$$
E_{E V}=\sum_{k=1}^{N} P_{E V_{-} k} \Phi_{k} T_{D}+\sum_{l=1}^{M} P_{E V_{-}-} \Phi_{l} T_{B}
$$

iv. The SAC with highest efficiency difference $\bar{\eta}_{\text {Hybrid }}-\eta_{E V}$ is chosen with hybrid operation and the required energy $E_{E V}$ will be updated based on Eq.(16), where $P_{\text {Hybrid }}$ and $P_{E V}$ are the battery power of the Hybrid and EV mode, $\Phi$ is the probability distribution for that SAC.

$$
E_{E V_{-} \text {new }}=E_{E V}+P_{H y b r i d} \Phi T_{D}-P_{E V} \Phi T_{D}
$$


v. Step iv is repeated until $E_{E V}$ is less than or equal to $E_{a v}$. If after looping through all SAC and $E_{E V}$ is still greater than $E_{a v}$, it indicates that the current sizing is not capable of finishing the cycle and it will be marked as an infeasible design.

After determining the hybrid operation mode, the PFC is calculated from Eq. (17), where the total number of Hybrid operation is dented as $n_{H}$.

$$
P F C=\sum_{i=1}^{n_{H}} \text { fuel }_{i} \Phi_{i} T_{D}
$$

Step 4:

Step 2 and Step 3 are repeated for selected sizing parameters, until all sizing parameters are looped through. The design with the lowest PFC is marked as the optimal.

\section{Designing Power-Split Hybrid Vehicles Using PEARS METHOD}

In this section, we first analyze the effectiveness of the PEARS by comparing it with the DP result with one design dimension. Then, the proposed rapid sizing method is applied to all input-split configurations of single-PG hybrid with multiple operation modes. DP will be used to verify fuel economy performance and the result will be compared with initial design with Prius' parameter shown in Table 1.

\section{A. Validation of the PEARS method}

A reliable sizing method should be able to match up with the result executed by optimal control strategy. The PFCs from the PEARS method are plotted in Figure 8 for FUDS cycle, with Final drive Ratio (FR), R:S ratio and MG sizes as the design variables. The initial parameters shown in Table 1 are from the Prius.
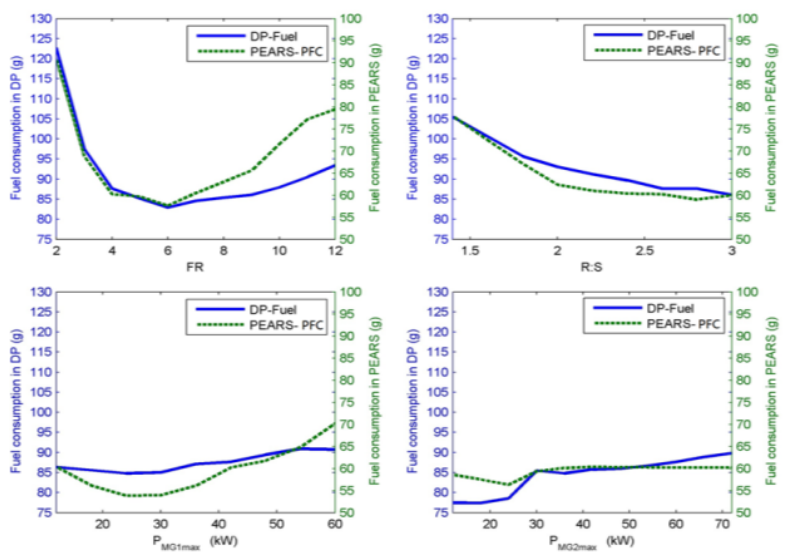

Figure 8. PFC and DP results over the FUDS cycle

It can be seen from Figure 8 that the results from the two methods are very similar, indicating that the PEARS method can be used for the sizing study.

Table 1 Design Variables to Verify the Validity of Pears Method

\begin{tabular}{|l|c|c|c|c|}
\hline \multirow{2}{*}{} & \multicolumn{4}{|c|}{ Design Variables } \\
\cline { 2 - 5 } & $F R$ & $R: S$ & $P_{\text {MGImax }}(\mathrm{kW})$ & $P_{M G 2 \max }(\mathrm{kW})$ \\
\hline Initial design & 4 & 2.6 & 42 & 60 \\
\hline Range & $2: 1: 12$ & $1.4: 0.2: 3.0$ & $12: 6: 60$ & $12: 6: 72$ \\
\hline
\end{tabular}

\section{B. Case study}

All six input-split configurations studied are shown in Figure 9, where the first one is similar to Prius (the original Prius doesn't have any clutch).
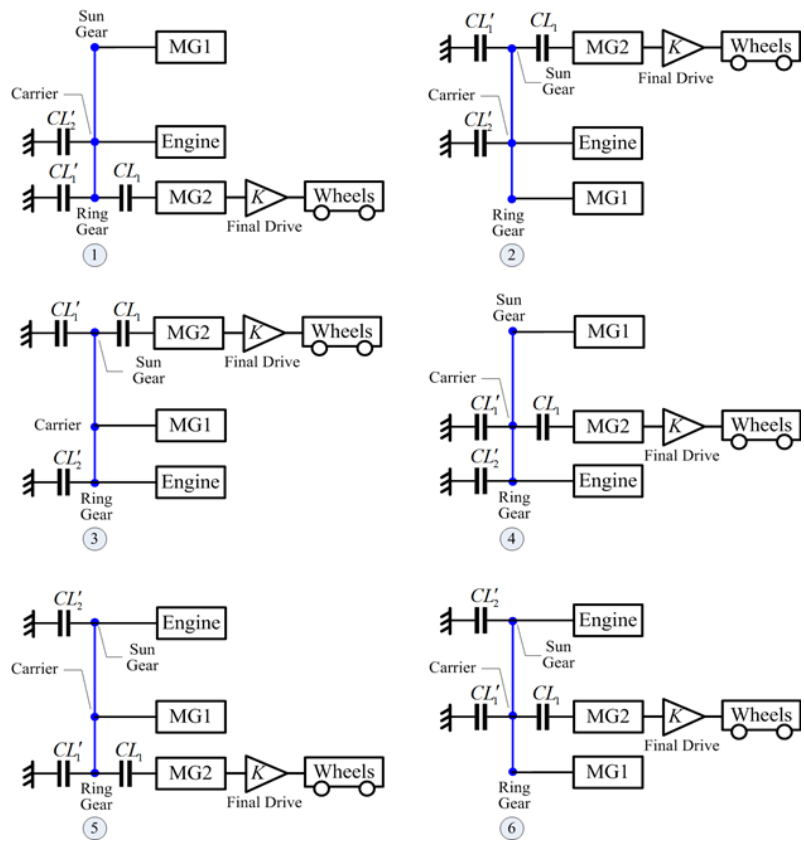

Figure 9. Six input-split configurations with single planetary gear

To exam the effectiveness of the PEARS method, the Prius parameters are used as initial parameters for all 6 input-split configurations. There are four design variables in total. In this step FR is kept within a practical range of 2:0.5:6, whereas the $\mathrm{R}: \mathrm{S}$ ratio and the sizing pool of MG1 and MG2 remain the same as the design range in Table 1.

Fuel Consumption in FUDS Cycle

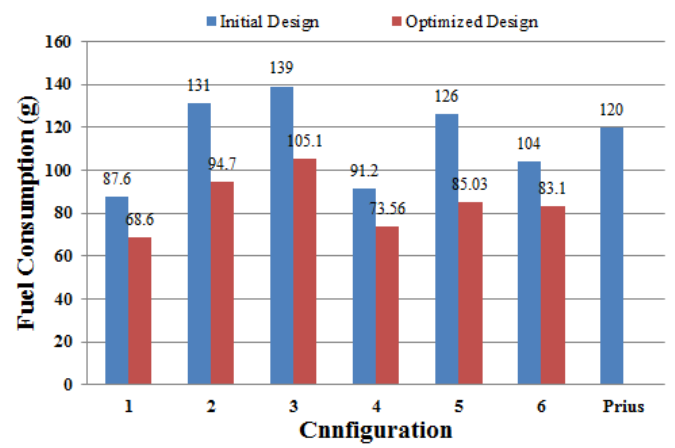

Figure 10. Fuel economy comparison between the initial design and optimized design in FUDS cycle

The optimal parameters and fuel consumption from DP are presented in Table 2. Note that the fuel consumption is calculated with the SOC drops from 0.55 to 0.43 to enable both hybrid and EV drive. The calculation time for each configuration in FUDS cycle with PEARS is around 8.3 hours, compared with the forecasted 88000 hours for DP, on a desktop with an i5-2500k 3.3 GHz CPU and 16G RAM.

The comparison between the initial sizing and optimized sizing are shown in Figure 10. It can be seen that significant fuel economy improvement could be achieved for all configurations on the FUDS Cycle. 
TABLE 2 OPTIMIZED DeSIGN PARAMETERS AND CORRESPONDING FueL CONSUMPTION IN FUDS CYCLE FOR PHEV

\begin{tabular}{|c|c|c|c|c|c|c|}
\hline \multirow{2}{*}{ Configuration } & \multicolumn{4}{|c|}{ Design Parameters } & \multicolumn{2}{c|}{ Fuel Consumption(g) } \\
\cline { 2 - 7 } & $F R$ & $R: S$ & $\begin{array}{c}P_{M G 1} \\
\begin{array}{c}\max \\
(\mathrm{kW})\end{array}\end{array}$ & $\begin{array}{c}P_{M G 2} \\
\text { max } \\
(\mathrm{kW})\end{array}$ & $\begin{array}{c}\text { Optimized } \\
\text { Sizing }\end{array}$ & $\begin{array}{c}\text { Initial } \\
\text { Sizing }\end{array}$ \\
\hline No.1 & 6 & 3 & 18 & 24 & 68.6 & 87.6 \\
\hline No.2 & 6 & 1.4 & 24 & 48 & 94.7 & 131.0 \\
\hline No.3 & 6 & 3 & 60 & 48 & 105.1 & 139.0 \\
\hline No.4 & 6 & 2 & 18 & 36 & 73.6 & 91.2 \\
\hline No.5 & 6 & 2.2 & 54 & 30 & 85.0 & 126.0 \\
\hline No.6 & 6 & 1.4 & 24 & 36 & 83.1 & 104.0 \\
\hline Prius & 4 & 2.6 & 42 & 60 & N/A & 120.0 \\
\hline
\end{tabular}

*: The fuel consumption is calculated with SOC dropping from 0.55 to 0.43 , during which about $3.2 \mathrm{MJ}$ battery energy is consumed. The mode shift NVH is not considered in this paper. **: The battery size of the Prius has been enlarged to enable plug-in feature and more EV drive

We also identify the optimal designs for HEVs, and found that in general they could be different from the optimal ones for PHEVs (see Table 3). This is because there are more EV operations in PHEVs.

TABle 3 Optimized Design PARAMETERs IN FUDS CyClE FOR HEV

\begin{tabular}{|c|c|c|c|c|}
\hline \multirow{2}{*}{ Configuration } & \multicolumn{5}{|c|}{ Design Parameters } \\
\cline { 2 - 5 } & $F R$ & $R: S$ & $\begin{array}{c}P_{\text {MGImax }} \\
(\mathrm{kW})\end{array}$ & $\begin{array}{c}P_{\text {MG2max }} \\
(\mathrm{kW})\end{array}$ \\
\hline No.1 & 6 & 3 & 12 & 18 \\
\hline No.2 & 6 & 1.4 & 30 & 36 \\
\hline No.3 & 6 & 1.8 & 54 & 42 \\
\hline No.4 & 4.5 & 3 & 18 & 36 \\
\hline No.5 & 6 & 1.4 & 60 & 24 \\
\hline No.6 & 3.5 & 1.4 & 36 & 60 \\
\hline
\end{tabular}

\section{CONCLUSION}

A rapid sizing method referred as Power-weighted Efficiency Analysis for Rapid Sizing (PEARS) is presented in this paper which is over 10,000 times faster than DP. The optimal sizing approach is based on power-weighted efficiency analysis and driving cycle information. Comparison with DP results confirms the validity of the proposed method. As a case study, all six input-split configurations using a single planetary gear as the transmission are analyzed for optimal sizing study. The optimization results show substantial improvement on fuel economy compared with initial parameters, which are those used in Toyota Prius. The optimization results indicate that it is possible to achieve fuel economy similar to the Prius using smaller electric machines. We should note however that other vehicle attributes, such as drivability and NVH, were not considered. We also confirmed that the optimal component sizing for PHEVs are different from those of HEVs.

The developed procedure can be applied to output-split configurations which is part of our current study. The PEARS strategy can also be applied to other vehicle designs, such as series hybrid, parallel hybrid and EV.

\section{ACKNOWLEDGMENT}

This material is based upon work supported by the Department of Energy under Award Number DE-PI0000012.

\section{REFERENCES}

[1] X. Zhang, C.Li, D.Kum, H. Peng, "Prius+ and Volt-: Configuration Analysis of Power-Split Hybrid Vehicles with a Single Planetary Gear", IEEE Transactions on Vehicular Technology, vol. 61, Issue 8, pp. 3544-3552, 2012.

[2] Alternative Fuels and Advanced Vehicles Data Center, Data, Analysis, and Trends: Vehicle—HEV Sales by Model, [online] 2010 http://www.afdc.energy.gov/afdc/data/vehicles.html (Accessed: $1 / 9 / 2012)$.

[3] B. Conlon, "Comparative analysis of single and combined hybrid electrically variable transmission operating modes," SAE Paper, 2005-01-1162, 2005.

[4] J. Liu and H. Peng, "A systematic design approach for two planetary gear split hybrid vehicles," Vehicle System Dynamics, vol. 48, pp. 1395-1412, 2010.

[5] C.-T. Li and H. Peng, "Optimal configuration design for hydraulic split hybrid vehicles," in American Control Conference, Baltimore, MD, 2010, pp. 5812-5817.

[6] M. Schmidt, "Two-mode, split power, electro-mechanical transmission," U.S. Patent 5577 973, 1996.

[7] X. Ai and S. Anderson, "Two-mode, compound-split, vehicular transmission having both enhanced speed tractive power," U.S. Patent 2006/0111212 A9, 2000.

[8] M. Raghavan, N. Bucknor and J. Hendrickson, "Electrically variable transmission having three interconnected planetary gear sets, two clutches and two brakes," U.S. Patent 7179 187, 2007

[9] J. Miller, "Propulsion system for hybrid vehicles", ISBN-10:0863413366, 2004.

[10] L. Husain, "Electric and hybrid vehicles", ISBN-10:143981175X, 2010.

[11] M. Kim, H. Peng, "Power management and design optimization of fuel cell/battery hybrid vehicles", Journal of Power Sources, vol. 165, Issue 2, 2007.

[12] D. Hermance, "Toyota Hybrid System," in SAE TOPTEC Conference, Albany, NY, 1999.

[13] N. Jalil, N. Kheir and M. Salman, "A rule-based energy management strategy for a series hybrid vehicle," in American Control Conference, Albuquerque, New Mexico, pp. 689-693, 1997.

[14] G. Pagalelli, S. Delprat, T. Guerra, J. Rimaux and J. Santin, "Equivalent consumption minimization strategy for parallel hybrid powertrains," in 55th IEEE Vehicular Technology Conference, 2002, pp. 2076-2081.

[15] A. Sciarretta, M. Back and L. Guzzella, "Optimal control of parallel hybrid electric vehicles," IEEE Transactions on Vehicular Technology, vol. 12, pp. 352-363, 2004.

[16] S. Delprat, J. Lauber, T. M. Guerra and J. Rimaux, "Control of a parallel hybrid powertrain: optimal control," IEEE Transactions on Vehicular Technology, vol. 53, pp. 872-881, 2004.

[17] S. Delprat, T. M. Guerra and J. Rimaux, "Control strategies for hybrid vehicles: optimal control," in 56th IEEE Vehicular Technology Conference, Vancouver, Canada, 2002, pp. 1681-1685.

[18] N. Kim, S. Cha and H. Peng, "Optimal control of hybrid electric vehicles based on Pontryagin's minimum principle," IEEE Transactions on Control Systems Technology, vol. 19, pp. 1279 - 1287, 2010.

[19] C.-C. Lin, H. Peng, J. Grizzle and J.-M. Kang, "Power management strategy for a parallel hybrid electric truck," IEEE Transactions on Control Systems Technology, vol. 11, pp. 839-849, 2003.

[20] J. Liu and H. Peng, "Control optimization for a power-split hybrid vehicle," in American Control Conference, Minneapolis, Minnesota, pp. 466-471, 2006.

[21] X. Zhang, C.Li, D. Kum, H. Peng, J. Sun, "Configuration Analysis for Power Split Hybrid Vehicles with Multiple Operating Modes", in AVEC Conference, Seoul, Korea, 2012. 